\title{
Comportamento da Pressão Arterial em Filhos de Normotensos e Filhos de Hipertensos Submetidos a Estímulos Pressóricos
}

\author{
José Wilson S. Cavalcante, Leonardo P. Cavalcante, Walewska S. Pacheco, Marcus G. F. de Menezes, \\ Carlos G. Gama F \\ Manaus, AM
}

Objetivo - Comparar a pressão arterial (PA) basal e as respostas a estímulos pressóricos de filhos de normotensos e filhos de hipertensos.

Métodos - Foram examinados 32 adolescentes, do sexo masculino, brancos, na faixa etária entre 13 e 18 anos, sendo que 16 eram filhos de hipertensos e 16 filhos de normotensos. Para cada indivíduo foi aferida a PA basal seguida da aplicação de três testes pressóricos: teste do exercício isométrico com o handgrip, teste pressor ao frio e teste do exercício aritmético mental. Para a aferição da PA foi utilizado um dispositivo oscilométrico, digital, previamente calibrado.

Resultados - Os filhos de hipertensos exibiram valores basais de PA, tanto sistólica quanto diastólica, maiores que os filhos de normotensos $(p<0,10)$. Quanto aos testes pressóricos, as respostas foram maiores nos filhos de hipertensos, porém, somente a resposta da PA diastólica ao teste do exercício aritmético mental alcançou significância estatística $(p<0,10)$.

Conclusão - Os resultados obtidos indicam a necessidade de atenção especial às crianças e adolescentes com antecedentes familiares de hipertensão.

Palavras-chave: testes pressóricos, adolescentes, fator genético

\section{Arterial Blood Pressure Responses in Chil- dren of Normotensive and Hypertensive Parents Submitted to Pressor Tests}

Purpose - To compare cardiovascular reactivity in adolescents with and without family history of hypertension.

Methods - Thirty two subjects, males, students, aging between 13 to 18 years old were examined. One half of them had no family history of hypertension and the other half had at least one hypertensive parent. Basal blood pressure level was obtained before the application of the following selected tests: isometric handgrip exercise test, cold pressor test and arithmetic mental stress test. The blood pressure values were obtained by a digital, oscilometric device, previously calibrated.

Results - The subjects with family history of hypertension exhibited higher basal levels of both systolic and diastolic blood pressure $(p<0,10)$. Concerning the tests applied, the subjects with family history of hypertension also exhibited higher responses, but only the response of the diastolic blood pressure to the mental stress test was statistically significant $(p<0,10)$.

Conclusion - Ourfindings point to the need of a special follow-up of children with a family history of hypertension.

Key-words: pressor tests, adolescents, genetic factor

Arq Bras Cardiol, volume 69 (n 5), 323-326, 1997

A hipertensão arterial (HA) é uma enfermidade que acomete cerca de $15 \%$ da população adulta brasileira ${ }^{1}$, com tendência a elevar sua prevalência com o aumento da faixa

Universidade do Amazonas - Manaus/CNPq

Correspondência: José Wilson S. Cavalcante - Av. Darcy Vargas, 378 - P. 10 69050-020 - Manaus, AM

Recebido para publicação em 2/12/96

Aceito em 16/9/97 etária $^{2}$. Representa um importante problema sob o ponto de vista de saúde pública, uma vez que compromete o sistema cardiovascular e a função renal, levando o indivíduo ao afastamento temporário ou definitivo de suas atividades laborativas e acarretando grandes repercussões sociais e econômicas ${ }^{3}$.

Considerando a problemática, estudos epidemiológicos têm sido realizados visando estudar o comportamento da pressão arterial (PA) em faixa etária mais precoce, 
procurando identificar os possíveis fatores condicionantes do desenvolvimento da HA ${ }^{4-6}$.

Na última década, tem crescido o interesse em se estudar o comportamento da PA de crianças e adolescentes submetidos a estímulos que induzem a elevação da $\mathrm{PA}^{7,8}$.

O presente trabalho analisa a diferença do comportamento da PA em filhos de indivíduos hipertensos e normotensos, em condições de repouso e mediante estímulos fisiológicos capazes de provocar elevação da PA, objetivando, diante dos resultados, propor condutas especiais para crianças e adolescentes que apresentam antecedente familiar de HA.

\section{Métodos}

Participaram da pesquisa 32 adolescentes na faixa etária de 13 a 18 anos, do sexo masculino, brancos, estudantes, filhos de normotensos e filhos de hipertensos, todos com índice de massa corporal (IMC) $<25$ e hábitos alimentares semelhantes, clinicamente saudáveis e residentes em Manaus (tab. I). A pesquisa foi realizada na Seção de Saúde do Colégio Militar de Manaus, com o consentimento, por escrito, dos alunos envolvidos na pesquisa e/ou seus responsáveis ${ }^{9}$. Foram incluídos, como filhos de hipertensos, aqueles que possuíam, pelo menos, um dos pais hipertensos.

Os testes pressóricos consistiram em: teste do exercício aritmético mental: a) com o indivíduo em decúbito dorsal horizontal, aferia-se sua PA e freqüência cardíaca (FC) no braço não dominante, após $10 \mathrm{~min}$ de repouso; b) solicitavase ao indivíduo realizar, mentalmente, rápida operação matemática, padronizada, durante $1 \mathrm{~min}$ : subtração de 7 em 7 a partir do número 100; c) aferia-se a PA e FC imediatamente após o término do estímulo, e aos 2, 4, 6, 8 e 10min subseqüentes; teste pressor ao frio: a) com o indivíduo em decúbito dorsal horizontal, aferia-se sua PA e FC no braço não dominante, após 10min de repouso; b) emergia-se a mão e antebraço (lado dominante) em uma cuba com água e gelo

\begin{tabular}{|lcc|}
\hline \multicolumn{2}{|c|}{$\begin{array}{c}\text { Tabela I - Distribuição dos filhos de normotensos e filhos de } \\
\text { hipertensos de acordo com a faixa etária }\end{array}$} \\
\hline Idade (anos) & Filhos de normotensos & Filhos de hipertensos \\
\hline $13-15$ & 9 & 5 \\
$16-18$ & 7 & 11 \\
\hline Total & 16 & 16 \\
\hline
\end{tabular}

\begin{tabular}{|lcc|}
\hline \multicolumn{3}{|c|}{$\begin{array}{c}\text { Tabela II - Comparação da pressão arterial basal de filhos de } \\
\text { normotensos e filhos de hipertensos }\end{array}$} \\
\hline & Sistólica* $(\mathrm{mmHg})$ & Diastólica* $(\mathrm{mmHg})$ \\
\hline \multirow{3}{*}{$\begin{array}{l}\text { Filhos de normotensos } \\
\text { Filhos de hipertensos }\end{array}$} & 98,94 & 53,27 \\
\hline p $<0,10$ & 103,48 & 56,42 \\
\hline
\end{tabular}

durante $1 \mathrm{~min}$; c) aferia-se a PA e FC imediatamente após o estímulo, e aos 2, 4, 6, 8 e 10min subseqüentes; teste do exercício isométrico com um handgrip: a) obtinha-se a força máxima do indivíduo, através da média de três medidas consecutivas; b) após $10 \mathrm{~min}$ de repouso, aferia-se a PA e FC; c) mantinha-se o handgrip em $30 \%$ da força máxima durante $3 \mathrm{~min}$; d) aferia-se a PA eFC imediatamente após o estímulo, e aos 2, 4, 6, 8 e 10min subseqüentes. Todas as aferições da PA eFC foram realizadas com dispositivo oscilométrico eletrônico, marca Sunbeam, modelo 7657, previamente calibrado.

Para análise estatística dos dados foi realizada análise de variância e, posteriormente, foi aplicado o teste estatístico Mann Whitney, para a comparação das médias dos valores pressóricos basais entre os dois grupos estudados e da média do percentual de elevação da PA (em resposta aos testes) entre os dois grupos.

\section{Resultados}

A média aritmética dos valores da PA medida antes de cada um dos três estímulos foi considerada basal. Esta média foi significativamente maior $(\mathrm{p}<0,10)$ nos filhos de hipertensos, tanto para a sistólica (PAS) quanto para a diastólica (PAD) (tab. II) e os resultados dos testes pressóricos encontram-se na tabela III.

No teste do exercício isométrico com o handgrip observou-se uma resposta pressórica maior ao estímulo, tanto da PAS quanto da PAD, no grupo de filhos de hipertensos, porém não houve significância estatística.

No teste pressor ao frio constatou-se maior resposta pressórica ao estímulo, tanto da PAS quanto da PAD, no grupo dos filhos de hipertensos, sem contudo alcançar significância estatística.

No teste do exercício aritmético mental observou-se maior resposta pressórica ao estímulo no grupo de filhos de hipertensos. Todavia, somente a resposta da PAD alcançou significância estatística $(\mathrm{p}<0,10)$.

Embora não tenham sofrido tratamento estatístico, os valores da PAS e PAD, obtidos durante os 10 min pós-estímulos mantiveram-se mais elevados nos filhos de hipertensos em todos testes (tab. IV).

\begin{tabular}{|c|c|c|c|}
\hline \multicolumn{4}{|c|}{$\begin{array}{l}\text { Tabela III - Percentual de crescimento da pressão arterial em resposta } \\
\text { aos estímulos pressóricos em filhos de normotensos (FNT) e filhos de } \\
\text { hipertensos (FHT) }\end{array}$} \\
\hline Teste & $\begin{array}{l}\text { Pressão } \\
\text { arterial }\end{array}$ & $\begin{array}{c}\text { FNT } \\
\text { (\% de cresc.) }\end{array}$ & $\begin{array}{c}\text { FHT } \\
\text { (\% de cresc.) }\end{array}$ \\
\hline $\begin{array}{l}\text { Exercício isométrico } \\
\text { com handgrip }\end{array}$ & $\begin{array}{l}\text { Sistólica } \\
\text { Diastólica }\end{array}$ & $\begin{array}{r}9,5( \pm 4,0) \\
9,1( \pm 15,3)\end{array}$ & $\begin{array}{r}12,4( \pm 5,6) \\
14,1( \pm 12,1)\end{array}$ \\
\hline Pressor ao frio & $\begin{array}{l}\text { Sistólica } \\
\text { Diastólica }\end{array}$ & $\begin{array}{l}17,7( \pm 11,8) \\
30,1( \pm 21,2)\end{array}$ & $\begin{array}{r}19,4( \pm 7,8) \\
34,6( \pm 32,3)\end{array}$ \\
\hline Aritmético mental & $\begin{array}{l}\text { Sistólica } \\
\text { Diastólica* }\end{array}$ & $\begin{array}{r}7,1( \pm 5,1) \\
13,6( \pm 19,1)\end{array}$ & $\begin{array}{r}7,7( \pm 6,0) \\
22,1( \pm 16,8)\end{array}$ \\
\hline
\end{tabular}




\begin{tabular}{|c|c|c|c|c|c|c|c|c|c|}
\hline Teste & & & Basal & Imediata & $2 \mathrm{~min}$ & $4 \mathrm{~min}$ & $6 \mathrm{~min}$ & $8 \min$ & $10 \mathrm{~min}$ \\
\hline \multirow{4}{*}{$\begin{array}{l}\text { Exercício isométrico } \\
\text { com o handgrip }\end{array}$} & FNT & PAS & 98,8 & 108,2 & 102,6 & 100,3 & 99,0 & 100,5 & 98,4 \\
\hline & & PAD & 56,0 & 60,3 & 56,3 & 55,0 & 54,1 & 54,3 & 52,5 \\
\hline & FHT & PAS & 103,0 & 116,0 & 106,5 & 104,8 & 104,1 & 105,0 & 104,5 \\
\hline & & PAD & 57,2 & 65,3 & 59,3 & 55,3 & 57,5 & 55,8 & 54,5 \\
\hline \multirow{4}{*}{ Pressor ao frio } & FNT & PAS & 98,8 & 116,0 & 106,3 & 103,8 & 102,5 & 101,3 & 102,5 \\
\hline & & PAD & 54,2 & 69,9 & 56,3 & 51,7 & 54,0 & 50,6 & 54,3 \\
\hline & FHT & PAS & 102,7 & 123,1 & 109,3 & 107,0 & 104,3 & 103,1 & 105,3 \\
\hline & & PAD & 56,1 & 74,5 & 59,8 & 56,8 & 54,5 & 53,5 & 53,6 \\
\hline \multirow{4}{*}{$\begin{array}{l}\text { Exercício aritmético } \\
\text { mental }\end{array}$} & FNT & PAS & 99,1 & 105,8 & 102,8 & 103,4 & 101,2 & 101,8 & 101,5 \\
\hline & & PAD & 49,5 & 55,8 & 51,8 & 52,3 & 48,6 & 51,3 & 48,6 \\
\hline & FHT & PAS & 104,6 & 112,5 & 104,8 & 105,7 & 104,9 & 105,7 & 105,9 \\
\hline & & PAD & 55,5 & 67,7 & 56,8 & 56,3 & 57,7 & 56,0 & 56,0 \\
\hline
\end{tabular}

\section{Discussão}

Investigações epidemiológicas e pesquisas no campo da biologia molecular permitem, hoje, afirmar que o surgimento da HA primária está na dependência de fatores genéticos e ambientais, com a participação dos primeiros variando entre 30 a $60 \%{ }^{10}$.

A combinação de dados dos estudos de Framingham MA, Tecumseh MI, Evans County GA, Canadian Adoption e do National Heart, Lung and Blood Institute ${ }^{11}$, relativos à correlação da PA entre pessoas com diferentes graus de parentesco, assinalam uma fraca correlação entre cônjuges e entre pais e filhos adotivos, apesar de compartilharem dos mesmos hábitos. Entretanto, o índice de correlação duplicou-se entre pais e filhos consangüíneos, tornando-se maior entre gêmeos dizigóticos e, ainda muito maior, entre gêmeos monozigóticos. Estes importantes estudos evidenciam a forte influência genética no comportamento da PA. Caulfield e $\mathrm{col}^{12}$, recentemente, estudando as características genéticas de 63 famílias em que dois ou mais membros eram portadores de HA primária, demonstraram uma ligação forte e consistente da hipertensão com as alterações no gene do angiotensinogênio, localizado no cromossomo 1q42-43.

Os testes pressóricos como o pressor ao frio, o do exercício aritmético mental, o do exercício isométrico e o do exercício ergométrico têm sido considerados como procedimentos capazes de identificar pessoas com predisposição a desenvolverem HA ${ }^{8,13,14}$. Já há 60 anos, Hines e Brown ${ }^{15}$, utilizando o teste pressor ao frio, admitiram que uma resposta exacerbada ao estímulo poderia predizer o surgimento de HA no futuro. Wood e $\mathrm{Col}^{7}$, realizando um estudo de seguimento de 45 anos com os indivíduos originalmente investigados por Hines e Brown, utilizando a mesma metodologia, encontraram uma correlação positiva entre a resposta exacerbada ao estímulo pressor ao frio e o desenvolvimento de
HA. Estes autores verificaram, ainda, que a presença de história familiar de hipertensão entre os hiper-reativos foi significativamente maior que entre os indivíduos normorreativos. Fredrikson e Matthews ${ }^{16}$, através de meta-análise, demonstraram que indivíduos com hipertensão limítrofe apresentam maior resposta da PA e da FC aos testes pressóricos, quando comparados aos normotensos. Voors e $\mathrm{col}^{17}$, estudando a PA em crianças na faixa etária de 5 a 14 anos, em uma comunidade da cidade de Bogalusa, EUA, salientam que a história natural da HA primária não será compreendida até que a evolução dos níveis tensionais e as alterações precoces no sistema cardiovascular sejam reconhecidas, com a identificação das causas determinantes, incluindo a influência dos fatores familiares e ambientais.

Os dados já existentes nesta linha de pesquisa aventam a possibilidade de se prever, através de testes pressóricos e em estágio precoce da vida, as crianças e adolescentes que apresentam predisposição à HA na vida adulta. Nossos achados, embora oriundos de uma amostra pequena, associam-se aos encontrados na literatura. Os níveis mais elevados de PA basal, maior resposta aos estímulos pressóricos e maiores níveis da PA até os 10min pós-estímulo, encontrados nos filhos de hipertensos, quando comparados aos filhos de normotensos, estando ambos os grupos com IMC, hábitos alimentares e sociais semelhantes, indicam a necessidade de atenção especial no acompanhamento das crianças e adolescentes com antecedente familiar de hipertensão. A eles devemos, com maior ênfase, recomendar exercícios aeróbicos, evitar a ingestão excessiva de alimentos com alto teor de sal e manter o IMC em níveis normais.

\section{Agradecimentos}

Ao Prof. João Caldas, do Instituto de Ciências Exatas da Universidade do Amazonas, pela realização da análise estatística. 


\section{Referências}

1. II Consenso Brasileiro para o Tratamento da Hipertensão Arterial. Arq Bras Cardiol 1994; 63: 333-47.

2. Cavalcante JWS, Daza CMG, Cavalcante LP et al. - Prevalência e aspectos socioculturais e econômicos da hipertensão arterial em Centro de Saúde da Zona Norte de Manaus. Arq Bras Cardiol 1995; 65: 493-6.

3. Doenças Cardiovasculares no Brasil - Sistema Único de Saúde - SUS - Dados Epidemiológicos - Assistência Médica. Brasília, Ministério da Saúde. Coordenação de Doenças Cardiovasculares, 1993.

4. Cavalcante JWS - Estimativa da pressão arterial em crianças por equação de regressão. Arq Bras Cardiol 1989; 52: 197-200.

5. Cavalcante JWS - Estudo epidemiológico da hipertensão arterial em crianças (tese de Mestrado em Cardiologia). Rio de Janeiro: UFRJ, 1976; 61p.

6. Brandão PA - A importância do desenvolvimento físico no comportamento da curva de pressão arterial em crianças de 6 a 9 anos de idade. Arq Bras Cardiol 1987; 48: 203-9.

7. Wood DL, Sheps SG, Elveback LR, Schirger A - Cold pressor test as a predictor of hypertension. Hypertension 1984; 6: 301-6.

8. ParkerF, Croft J, Cresenta J et al - The association between cardiovascular response tasks and future blood pressure levels in children: Bogalusa Heart Study. Am Heart J 1987: 113: 1174-9.
9. Conselho Nacional da Saúde - Resolução 01/88: Normas de Pesquisa em Saúde. Diário Oficial da União 1988: 1073-119.

10. Swales JD-Manual of Hypertension. Londres: Blackwell Science Ltda, 1995: 39.

11. Kaplan NM - Clinical Hypertension. $5^{\text {th }}$ ed. Maryland: Williams \& Wilkins, 1990: 55

12. Caulfield M, Lavender P, Farral M et al - Linkage of the angiotensinogen gene to essential hypertension. N Engl J Med 1994; 330: 1629-33.

13. Falkner B, KushnerH, Onesti G Angelakos ET - Cardiovascular characteristics in adolescents who develop essential hypertension. Hypertension 1981; 3: $521-7$.

14. Herkenhoff FL, LimaEG, VasquezEC, Mill JG-Importância dos testes de estresse no diagnóstico da hipertensão arterial. Arq Bras Cardiol 1994; 62: 439-43.

15. Hines JrEA, Brown GE-The cold pressor test for measuring the reactibility of the blood pressure: data concerning 571 normal and hypertensive subjects. Am Heart J 1936; 11: 1-9.

16. Fredrikson M, Matthews KA-Cardiovascular responses to behavioral stress and hypertension: A meta-analytic review. Ann Behav Med 1990; 12: 30-9.

17. Voors AW, Foster TA, Frerichs RR, Webber LS, Berenson GS - Studies of blood pressure in children, ages 5-14 years, in a total biracial community: the Bogalusa Heart Study. Circulation 1976; 54: 319-27. 